We sought to determine if our unit was failing to identify neonates at risk of HDFN who were born to RhD positive mothers with negative antibody screening at booking who developed antibodies later during their pregnancy.

Methods We carried out a retrospective chart review of babies born to women who booked with a negative antibody screen who subsequently produced a clinically significant antibody in that pregnancy, from 2011 to 2017 inclusive.

The development of antibodies was detected incidentally via repeat maternal sampling or via repeat maternal testing following a positive Direct Coomb's test on their baby.

Results We identified 18 babies born to Rhesus D positive women who produced at least 1 of 8 clinically significant antibodies after their booking bloods were taken. 2 women tested positive for 2 antibodies. Any pregnancy where an immune Anti-D was produced was excluded.

$2 / 3$ rd of the at-risk infant population were male and $1 / 3 \mathrm{rd}$ female.

All tested DCT positive $14 \%$ of at-risk babies in the study group were diagnosed with jaundice;

2 due to Anti-E antibodies,

2 due to Anti-c antibodies \&

1 each due to Anti-Jka, - Jka \& -Jk3, Anti-Wra and AntiCw antibodies.

3 jaundiced neonates had no treatment.

4 needed phototherapy, of whom 2 also needed top-up red cell transfusion.

1 of these infants was later identified later as having Hereditary Spherocytosis accounting for his marked haemolysis and transfusion requirement.

Discussion Based on the retrospective review of this small cohort of neonates in a single centre, a lack of routine repeat screening for their RhD positive mothers did not increase the risk of HDFN-associated morbidity nor mortality.

We do not recommend routine repeat screening of Rhesus positive mothers for clinically-significant red cell allo-antibodies at 28-32 weeks in the absence of concern regarding haemolytic anaemia or jaundice in their babies. This would also confer a further 9,000 samples per year in our unit at a significant financial \& workload cost with no evident gain for our neonatal population.

\section{GP19 METHOTREXATE NEUROTOXICITY: MRI CHANGES IN CHILDREN WITH ALL}

Anne Haddick*, Anthony McCarthy, Christine Macartney. Royal Belfast Hospital for Sick Children, Belfast, UK

\subsection{6/archdischild-2019-epa.86}

Background Acute lymphoblastic leukaemia (ALL) is the commonest childhood malignancy in Europe. The survival rate has dramatically increased due to advances in treatment and it is of extreme importance to optimise and individualise treatment for each child. A crucial drug in this treatment is methotrexate. This can be given through the oral, intravenous and intrathecal route. However, methotrexate has various side effects, particularly neurotoxicity. MRI typically shows white matter changes known as leukoencephalopathy. The aim of this study was to look at the MRI changes in these patients and to determine persistence of neurological effects.

Method A retrospective study looking at patients, treated for ALL, on UK ALL 2003 and 2011 trials in a single centre who developed Methotrexate Neurotoxicity were selected. Parameters including type of symptoms, length of symptoms, and timing from last Methotrexate dose were correlated with MRI findings. Results There were 6 patients out of 138 enrolled on study who were diagnosed with neurotoxicity following MTX treatment. The age ranged from 4-11 years old with a mean age of 7. All 6 patients had MRIs. 2 patients had changes on their scans. White matter changes were displayed consistent with leukoencephalopathy. Both patients have been followed up in clinic and have no further neurological deficit. There have been no reports of further neurological deficit in the other 4 patients. Novel findings in this study found that patients with MRI changes also showed generalised changes on their EEG.

Conclusions This study describes the clinical course and radiological findings of patients with Methotrexate-induced Leukoencephalopathy. This appears to be a reversible entity without any apparent long-term effects. Long term follow-up and evaluation will be necessary to confirm this. Prospective add-on studies are warranted to evaluate this issue in a more robust manner.

\section{GP20 RETINOBLASTOMA JOURNEY FROM A TERTIARY CENTER: FROM 1981 TO 2017}

Betül Çınar*, M.Alp Özkan, A.Murat Sarıı. Istanbul University Cerrahpaşa Medical Faculty, istanbul, Turkey

\subsection{6/archdischild-2019-epa.87}

Introduction Retinoblastoma is the most common intraocular tumor in childhood originating from sensory epithelium of retina. This tumor, which is seen one in 15,000 live births, has unilateral involvement in $2 / 3$ cases and bilateral involvement in $1 / 3$ cases. There is no difference between male and female cases in terms of gender. In the past, while primary therapy was enucleation, currently chemotherapy protocols and focal therapies are preferred. We aimed to obtain the changes in terms of enucleation rates over the years and compare survival data with literature.

Methods Patients diagnosed with retinoblastoma between 1981 and 2017 were enrolled in the study. Gender, laterality, age at diagnosis, initial complaint, spread of tumor, treatment modalities, enucleation rates, overall survival data of 221 patients obtained retrospectively. Vital status and missing medical data were achieved by phone calls. Regular physical examination, blood counts, systemic and local side effect evaluations performed every month before chemotherapy. Analysis was done using SPSS software for Windows 11.0. Survival rates were calculated by means of Kaplan-Meier and log-rank tests. Statistical significance was inferred at $\mathrm{p}<0.05$.

Results From 1981 to 2000, the enucleation rate was $96.9 \%$, after 2000 it decreased to $66.3 \%$. The rate of enucleation in ICRB(International Classification of Retinoblastoma) group E patients was $82.3 \%$. The rate of enucleation was higher in group $\mathrm{E}$ than in group D. Overall 5 -year survival was $88 \%$. This rate was $98 \%$ for unilateral cases and $72 \%$ for bilateral cases. Overall survival in metastatic patients was $56.4 \%$. Fifteen cases were lost in follow-up. Bilaterality and extraocular presentation at first were found to be inversely proportional to survival, while sex and age did not differ significantly.

Conclusion Retinoblastoma survival rates are gradually increasing with widely used focal and systemic therapies. Innovations in retinoblastoma treatment should focus not only on saving lives but also sparing the globe and vision. 